\title{
Positional irradiance measurement: characterization of spectrum- splitting and concentrating optics for photovoltaics
}

\author{
Cristofer A. Flowers ${ }^{*}$, Sunita Darbe ${ }^{\mathrm{a}}$, Carissa N. Eisler ${ }^{\mathrm{a}}$, Junwen $\mathrm{He}^{\mathrm{b}}$, Harry A. Atwater ${ }^{\mathrm{a}}$ \\ ${ }^{a}$ Watson Research Laboratories, California Institute of Technology, Pasadena, CA, USA 91125; \\ ${ }^{\mathrm{b}}$ Frederick Seitz Materials Research Laboratory, University of Illinois at Urbana-Champaign, \\ Urbana, IL, USA 61801
}

\begin{abstract}
Multijunction photovoltaics enable significantly improved efficiency over their single junction analogues by mitigating unabsorbed sub-bandgap photons and voltage loss to carrier thermalization. Lateral spectrum-splitting configurations promise further increased efficiency through relaxation of the lattice- and current-matching requirements of monolithic stacks, albeit at the cost of increased optical and electrical complexity. Consequently, in order to achieve an effective spectrum-splitting photovoltaic configuration it is essential that all optical losses and photon misallocation be characterized and subsequently minimized.

We have developed a characterization system that enables us to map the spatial, spectral, and angular distribution of illumination incident on the subcell reception plane or emerging from any subset of the concentrating and splitting optics. This positional irradiance measurement system (PIMS) comprises four motorized stages assembled in an X-Z-R$\mathrm{Y}$ configuration with three linear degrees of freedom and one rotational degree of freedom, on which we mount an optical fiber connected to a set of spectrometers covering the solar spectrum from 280-1700 nm. In combination with a xenon arc lamp solar simulator with a divergence half angle of 1.3 degrees, we are able to characterize our optics across the full spectrum of our photovoltaic subcells with close agreement to outdoor conditions. We have used this tool to spectrally characterize holographic diffraction efficiency versus diffraction angle; multilayer dielectric filter transmission and reflection efficiency versus filter incidence angle; and aspheric lens chromatic aberration versus optic-to-receiver separation distance. These examples illustrate the versatility of the PIMS in characterizing optical performance relevant to both spectrum-splitting and traditional multijunction photovoltaics.
\end{abstract}

Keywords: photovoltaics, spectrum splitting, optical characterization, dichroic filter, hologram, aspheric lens, spectrometry

\section{INTRODUCTION}

Lateral spectrum splitting photovoltaics offer potential for unprecedented sunlight-to-electricity conversion efficiency. ${ }^{1-3}$ This is enabled by the facile pathway to a larger number of junctions ${ }^{4}$, the wider material selection with reduced lattice matching constraints relative to monolithic multijunction photovoltaics, and improved response to spectral variability with independent electrical connection to each of the subcells. ${ }^{5}$ Despite these advantages, no spectrum-splitting embodiment to date has surpassed the record efficiency of monolithic multijunction devices. Recent interest in spectrum splitting has focused on novel optical design methods and configurations to enable improved form factors and increased efficiency. ${ }^{6-9}$

Fundamentally, spectrum splitting optics require precise characterization of the laterally dispersed illumination incident on each subcell, as well as any stray light lost to passive elements within the photovoltaic system. We have developed an optical characterization system that enables us to map the spatial, spectral, and angular distribution of illumination incident on an optical reception plane. The tool combines four motorized stages for three-dimensional and rotational positioning control with a fiber optic-coupled spectrometer spanning 280-1700 nm. Proof of concept has been demonstrated by using the positional irradiance measurement system (PIMS) to characterize diverse spectrum splitting and concentrating optical elements: a transmission hologram, two bandpass dichroic filters, and a concentrating aspheric lens with chromatic aberration. Hologram measurements demonstrate goniospectral characterization with varying transmission as a function diffraction angle, dichroic filter measurements demonstrate spectral characterization with

* cflowers@,caltech.edu; phone 1626395 3983; daedalus.caltech.edu

High and Low Concentrator Systems for Solar Energy Applications IX, edited by Adam P. Plesniak, Candace Pfefferkorn, Proc. of SPIE Vol. 9175, 91750F - (c) 2014 SPIE · CCC code: 0277-786X/14/\$18 · doi: 10.1117/12.2062245 
varying transmission as a function of incidence angle, and aspheric lens measurements demonstrate one dimension of spatial-spectral characterization.

\section{EXPERIMENTAL}

\subsection{Positional irradiance measurement system}

The PIMS, shown in Figure 1, is designed to perform spatial-spectral mapping ${ }^{9}$ on a reception plane with incident angle resolution, producing a spatially resolved goniospectral map. The system consists of three linear and one rotational stages stacked in an X-Z-R-Y configuration, where the z-axis is the axis of optical propagation. The end of an optical fiber is mounted to the Y-stage in order to be rastered in in two dimensions to map the optical reception plane. Angular resolution is gained by placing an aperture at the front of the optical fiber to restrict the angular acceptance range and acquiring a spatial-spectral map at several angles of incidence by rotating the entire optical assembly about its y-axis. The optical fiber is trifurcated and transmitted light impinges onto three spectrometer pixel arrays to cover the spectral range of interest for spectrum splitting solar energy conversion.

Each of these capabilities is critical to properly predicting the system efficiency for a spectrum splitting photovoltaic device. The spectral response of a solar cell depends both on wavelength and angle of incidence, and an optimal antireflection (AR) coating will account for spectral and angular distribution. Beyond subcell illumination, validating spectrum splitting performance of the optical system requires knowledge of the input spectra to all optical elements arranged in parallel. Additionally, a thermodynamically optimal optical system will match the etendue between optical components connected in series. Characterizing the angular and spatial extent of the output of each optic in the system is necessary to properly design each subsequent optical element. This information also enables accounting for discrepancies between the performance of ideal components and as-fabricated components for subsequent design iterations.

\section{RESULTS AND DISCUSSION}

\subsection{Transmission hologram}

The PIMS was used to characterize the diffractive properties of a transmission hologram designed to perform spectrum splitting in the configuration pictured in Figure 2a. In this design, stacked sets of holograms diffract normally incident illumination into frequency bands directed onto underlying subcells. Concentration is performed after spectrum splitting as the holograms exhibit relatively low tolerance to incident angular spread. Subcell bandgaps are each optimized for conversion of the resulting incident spectrum.

The experimentally characterized hologram was purchased off-the-shelf from Wasatch Photonics, and an in-house analytical model was used to predict the diffraction efficiency across the solar spectrum. The hologram was held fixed perpendicular to the light path while the detector was swept in $\theta$ at constant separation distance, and a spectrum was collected at each diffraction angle. An aperture was placed at the front of the hologram to eliminate edge effects. A second aperture was placed in front of the collection fiber to limit the collected angular spread to approximately one degree. Incident illumination from a Xenon arc-lamp solar simulator with a divergence half angle of $1.3^{\circ}$ was used for experimental characterization, while a collimated beam with incidence angle of $0.5^{\circ}$ off-normal was assumed in analytical modeling. Data were simulated and collected in one degree increments.

Diffraction efficiency, the ratio of intensity in a diffracted order to intensity in the incident illumination, is plotted against wavelength and angle for analytical and measured data in Figures $3 a$ and $b$, respectively. The zero ${ }^{\text {th }}$ diffracted order is omitted for clarity. The data show strong qualitative agreement with the first and second diffracted orders occurring at the expected angle throughout the visible region of the spectrum. The trend of reduced diffraction efficiency with increasing wavelength is also consistent between the data. A peak broadening due to the finite size of the collection aperture and the angular spread of the incident beam is observed in the experimental data and accounts for some of the quantitative discrepancy observed between peak diffraction efficiency in the data. Additionally, Fresnel reflections at the front and rear surfaces of the holographic optic and volume UV absorption, not accounted for in the analytical model, account for a portion of the numerical discrepancy between the data. In spite of this, the data definitively demonstrate the capability for angularly resolved characterization of spectrum splitting optics. Further refinement of the analytical calculation technique to incorporate features present in the experimental system is expected to produce excellent quantitative agreement with the experimental data. 


\subsection{Dichroic filters}

Dichroic filters are an additional optical element of interest for spectrum splitting. One embodiment is shown in Figure $2 \mathrm{~b}$, wherein individual frequency bands are sequentially transmitted to optimal bandgap subcells as remaining bands are reflected at approximately $90^{\circ}$ angles along the parallelepiped stack. A small amount of concentration is tolerable prior to splitting, while significantly more concentration can be incorporated after the filters. This is due to the transmission mechanism of multilayer dielectric stacks. The band cutoff shift with varying angle of incidence leads to poor performance under illumination with large angular extent.

It is challenging to extend the reflecting band of a shortpass filter out to $1700 \mathrm{~nm}$ while maintaining high performance elsewhere in the spectrum. Consequently, a high efficiency longpass filter with a cutoff at $1320 \mathrm{~nm}$ first transmits the furthest IR illumination onto a $0.73 \mathrm{eV}$ subcell before short pass filters transmit frequency bands sequentially from high to low energy.

A second configuration of the PIMS was used to characterize the transmitted spectrum as a function of incidence angle for the first two dichroic filters in this optical design. These $1320 \mathrm{~nm}$ longpass and $577 \mathrm{~nm}$ shortpass filters were designed in-house in collaboration with and fabricated by Chroma Technology. Each filter was mounted to the rotation stage of the PIMS, and variable incidence angle transmission data was obtained at $45 \pm 10^{\circ}$ angles of incidence in $5^{\circ}$ increments. The detector was held fixed in position during all measurements.

Forty-five degree incidence transmission data for the $1320 \mathrm{~nm}$ and $577 \mathrm{~nm}$ filters are shown in Figures $4 \mathrm{a}$ and $\mathrm{b}$, respectively. The filters are designed to be surrounded by an index 1.45 medium but are characterized in air. Normal incidence air-filter-air transmission is calculated and displayed for comparison as embedded performance is not obvious. There is strong agreement between peak position in design and experiment, with peak broadening due to the $1.3^{\circ}$ divergence half-angle of the illumination source. The $1320 \mathrm{~nm}$ filter has a power weighted average transmission of $97.0 \%$ in-band and $2.3 \%$ out-of-band, in agreement within $4.4 \%$ of the theory. The $577 \mathrm{~nm}$ filter has a power weighted average transmission of $96.2 \%$ in-band and $3.2 \%$ out-of-band, in agreement within $4.7 \%$ of the theory.

Off-design angle data for the $577 \mathrm{~nm}$ shortpass and $1320 \mathrm{~nm}$ longpass filters are shown in Figures $4 \mathrm{c}$ and d, respectively. The transmission band cutoffs blueshift and redshift at greater and lesser angles, respectively, while spectral features remain largely unaffected. This is precisely what would be expected due to the increased phase shift accumulation through each of the filter layers as the angle of incidence increases. The calculated power weighted error with respect to the theory ranges from 3.1-4.7\%, in good agreement with the design angle data and implying that the filters will perform as calculated off of the design angle as well. Collectively, these data demonstrate the ability to spectrally characterize transmission efficiency from optical elements while varying angle of illumination.

\subsection{Aspheric lens}

Concentrating optical elements are an essential component of nearly all terrestrial multijunction photovoltaics, including spectrum splitting devices. Imaging optics often suffer from chromatic aberration resulting from refractive index dispersion in the lens material. This phenomenon can be exploited for spectrum splitting by using small subcells separated by the difference in focal lengths between the wavelengths corresponding to the respective bandgaps of the subcells. One configuration utilizing this method of separation is pictured schematically in Figure 2c, wherein high and low bandgap cells would be placed in the focal planes of the blue and red light, respectively.

An aspheric lens from Edmund Optics was used as the concentrating optical element. The PIMS was used to characterize the focal length for several wavelengths and the corresponding spectral content at each focal length by scanning an aperture and detector along the Z-axis. The diameters of the aspheric lens and aperture were chosen to be $15 \mathrm{~mm}$ and $400 \mu \mathrm{m}$, respectively, such that unfocused or defocused wavelengths would not be collected by the optical fiber. Focal lengths from 400-1000 nm in $100 \mathrm{~nm}$ increments were determined by maximizing the collected intensity in the spectral window within $10 \mathrm{~nm}$ of each desired wavelength. In each case, only the z-position was adjusted while the $\mathrm{x}$ - and $\mathrm{y}$ positions and rotation were held fixed.

The ray-traced and experimentally measured focal lengths are plotted for each wavelength in Figure 5a. Chromatic aberration is more severe at the blue end of the spectrum, as expected from a Cauchy-like material. Spectra collected at each focal point and normalized to the incident spectrum are shown in Figure $5 \mathrm{~b}$ and compared to ray-traced values in Figure 5c. The data again show strong qualitative similarity, with close agreement in peak positions. The reduced optical efficiency at the blue end of the spectrum may be partially accounted for by a reduction in transmission efficiency 
through the optical fiber due to the increased angular spread required for concentration at shorter focal lengths and the approximate $\cos (\theta)$ transmission of the fiber coupler. Additionally, the discrepancy in the focal length simulation and experimental data suggest a slight angular misalignment of the optic relative to input illumination. This would place the collection aperture at the center of the focal spot for some wavelengths but slightly displaced in the xy-plane for others, in good agreement with the peak discrepancies observed between the experimental and simulated spectral profiles. These data demonstrate a one dimensional spatial-spectral mapping, and analysis of additional optical elements commonly used in the concentrating photovoltaics industry will expand the applicability.

\section{CONCLUSIONS}

The three optical element characterization measurements discussed provide a proof of concept and demonstrate the versatility of a multifunctional optical characterization tool for optics for photovoltaics. Characterization of an integrated optical assembly for concentration or spectrum splitting will require simultaneous mapping of the xy-plane spatially and angularly varying spectra across each subcell. This necessitates combination of linear and rotational movement of the collection fiber. An automated spectrum acquisition and raster of the collection fiber and apertures will be used to determine the spectral intensity distribution across all subcells under the polyhedral spectrum splitting optics. Acquisition of similar intensity distributions over a range of angles of incidence will be performed to facilitate optical component design validation and improvement, optimal AR coating design, and evaluation of other spectrum splitting and concentrating optical systems.

\section{ACKNOWLEDGEMENTS}

This work was supported by the Dow Chemical Company under the 'Full Spectrum Photovoltaics' project. J.H. and the aspheric lens optical design, simulation, and experimental characterization were supported by the DOE 'Light-Material Interactions in Energy Conversion’ Energy Frontier Research Center under grant DE-SC0001293. 


\section{FIGURES}

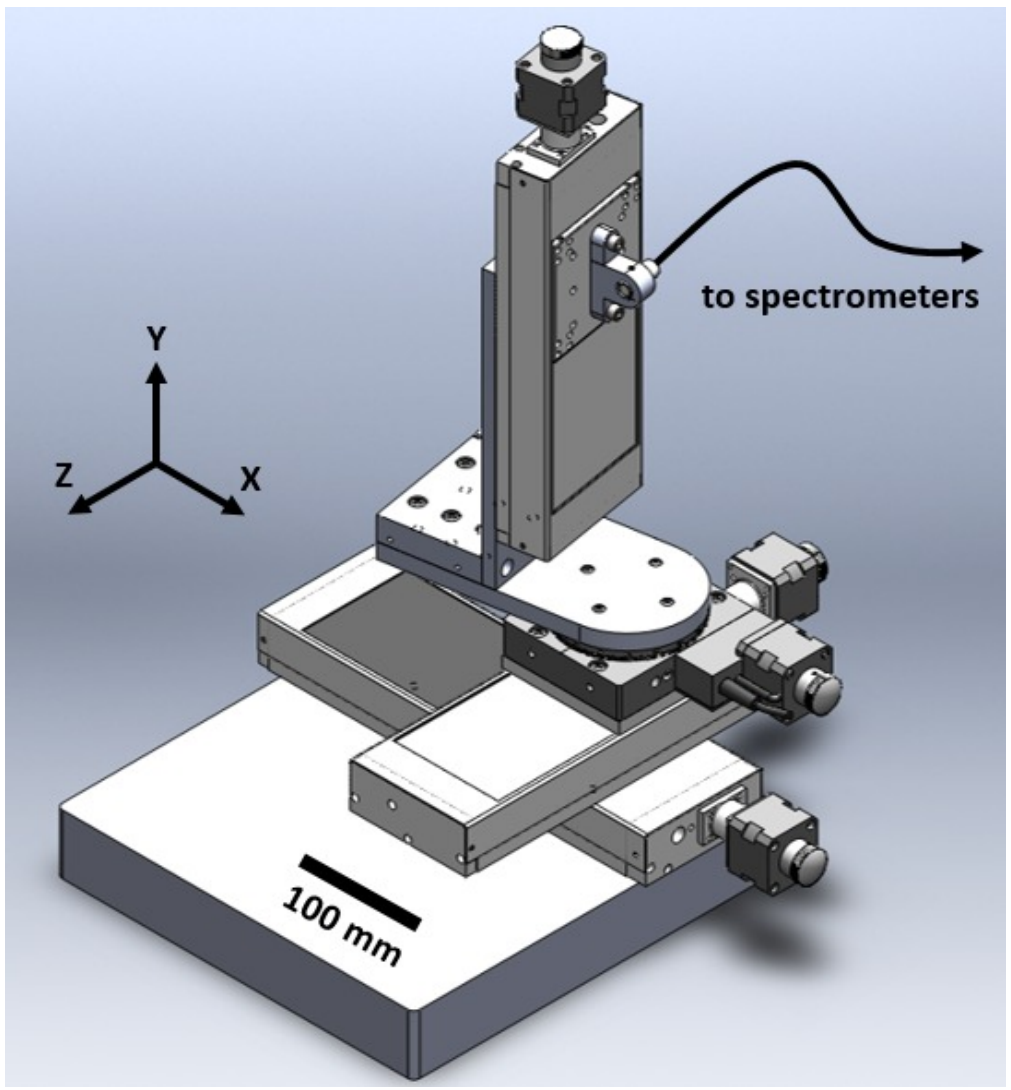

Figure 1: Positional irradiance measurement system solid model. The z-axis is the axis of optical propagation. The optical fiber tip is centered over the axis of rotation.

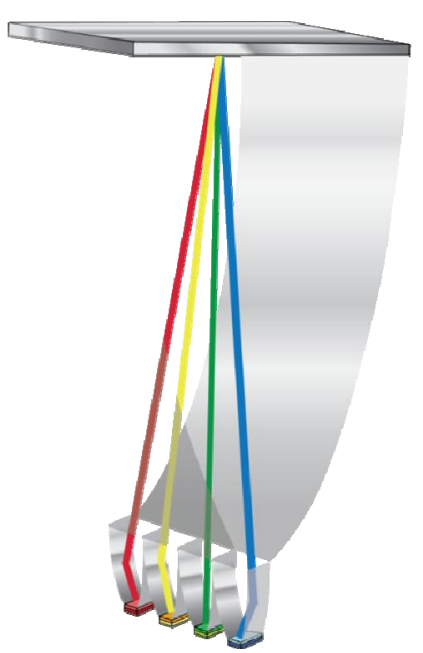

b.

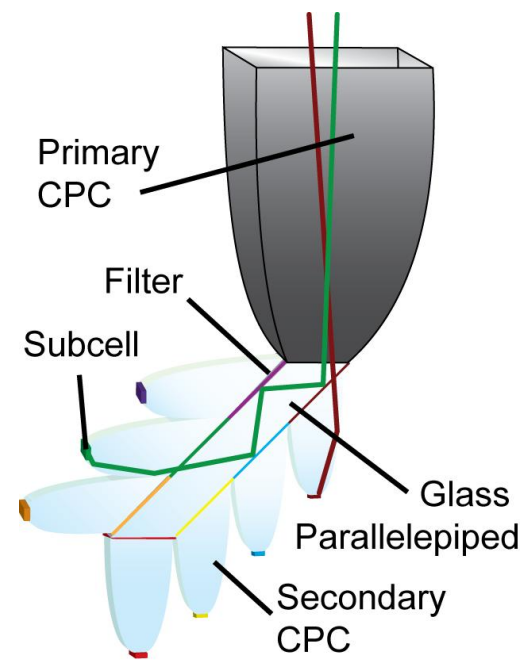

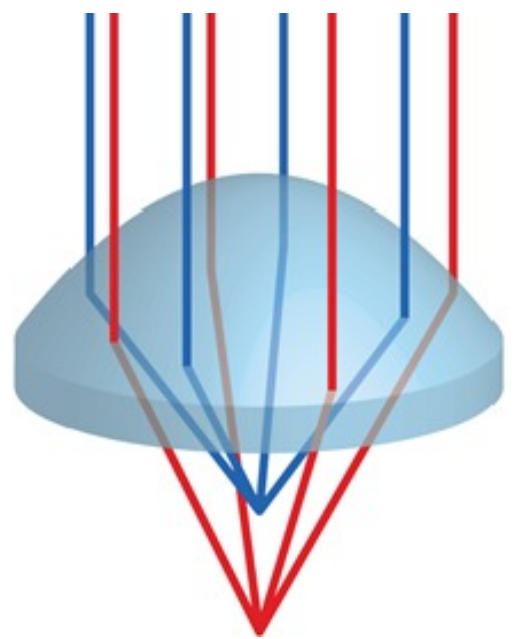

Figure 2: Schematics of optical systems under consideration: (a) transmission holographic spectrum splitting concentrator, (b) polyhedral specular reflector employing dichroic filters, and (c) aspheric lens with chromatic aberration. 
a.

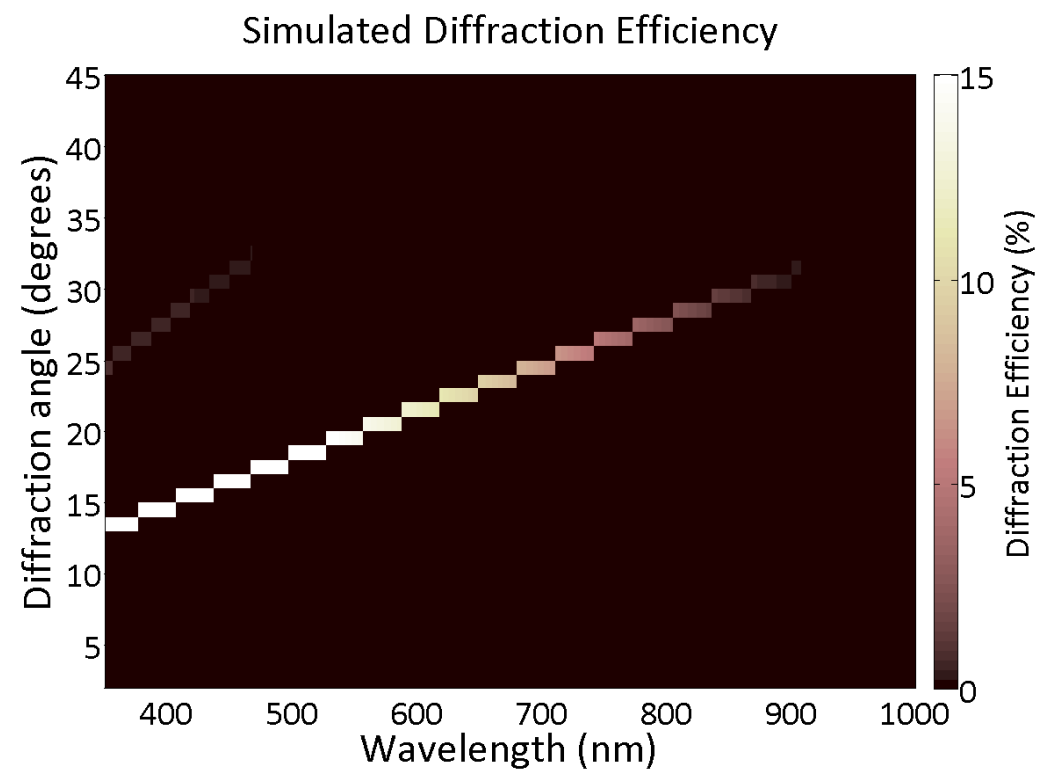

b.

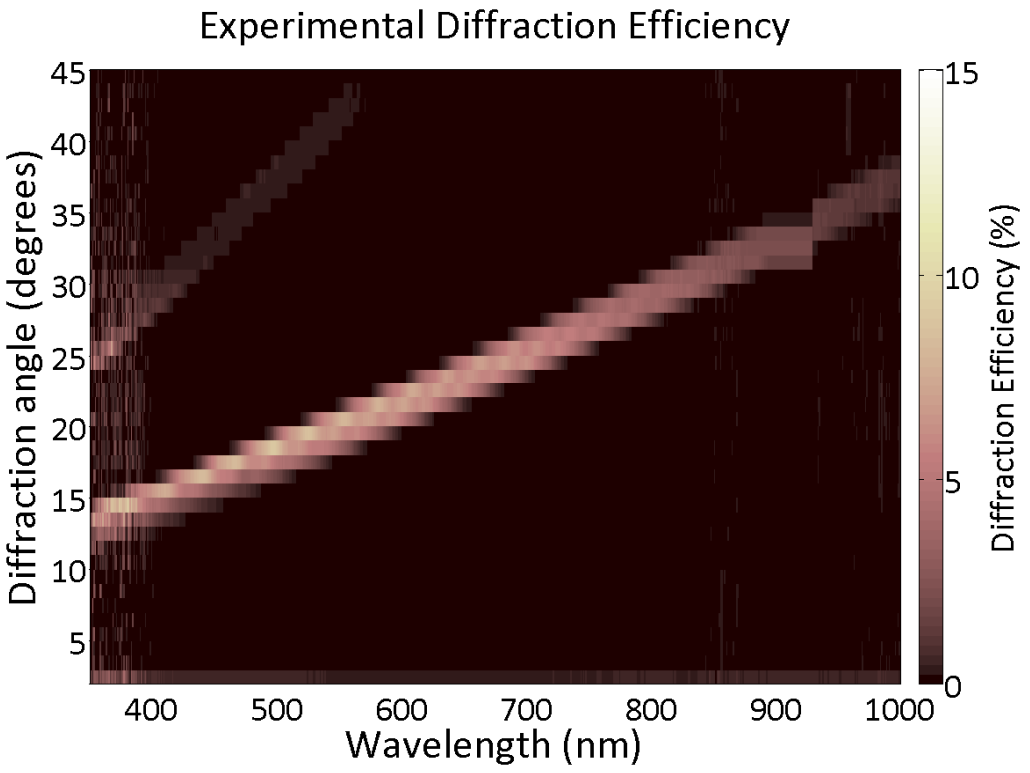

Figure 3: Diffraction efficiency of a transmission hologram in first and second order modes as a function of wavelength and angle. Analytical and measured data are shown in (a) and (b), respectively. 
a.

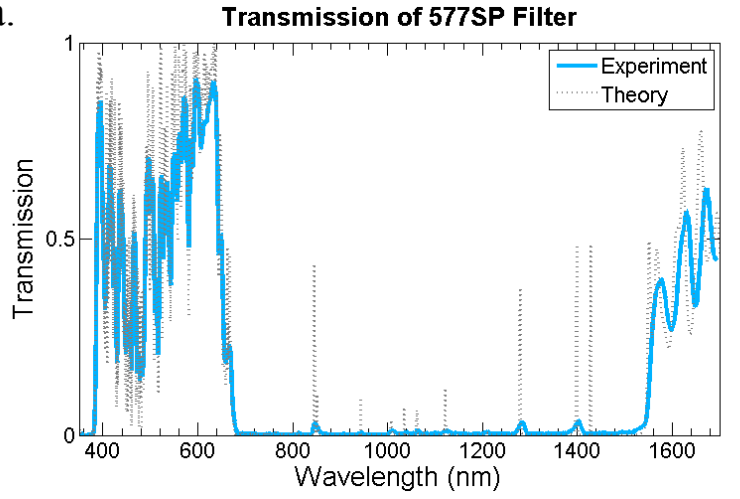

c. Experimental Transmission with Varied Angle of Incidence



b.

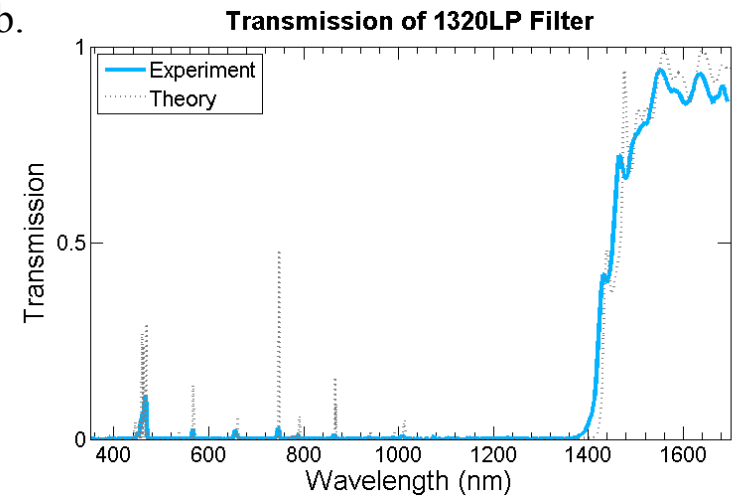

d. Experimental Transmission with Varied Angle of Incidence

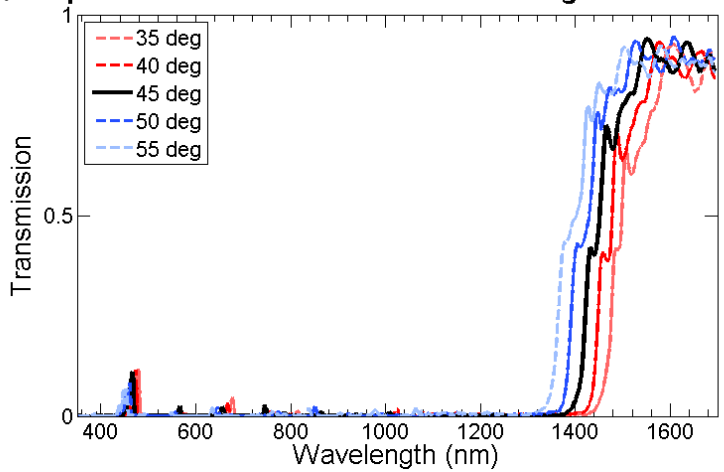

Figure 4: Theoretical and measured transmission performance of (a) $1320 \mathrm{~nm}$ longpass and (b) $577 \mathrm{~nm}$ shortpass dichroic filters in air, and angular response of the (c) $577 \mathrm{~nm}$ filter and (d) $1320 \mathrm{~nm}$ filter. Both filters were designed for glass encapsulation and match the design closely, with expected cutoff wavelength shift trend with changing angle of incidence. 
a.

\section{Focal Length Measurement}

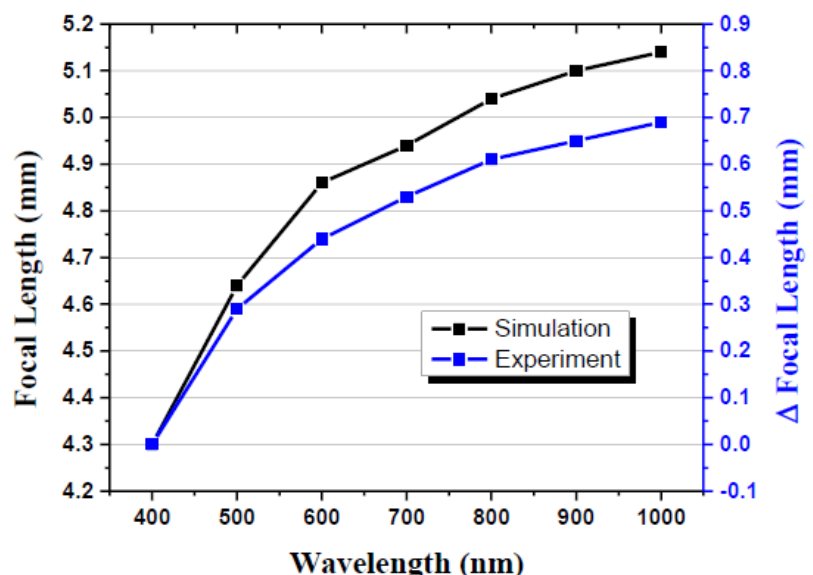

b.
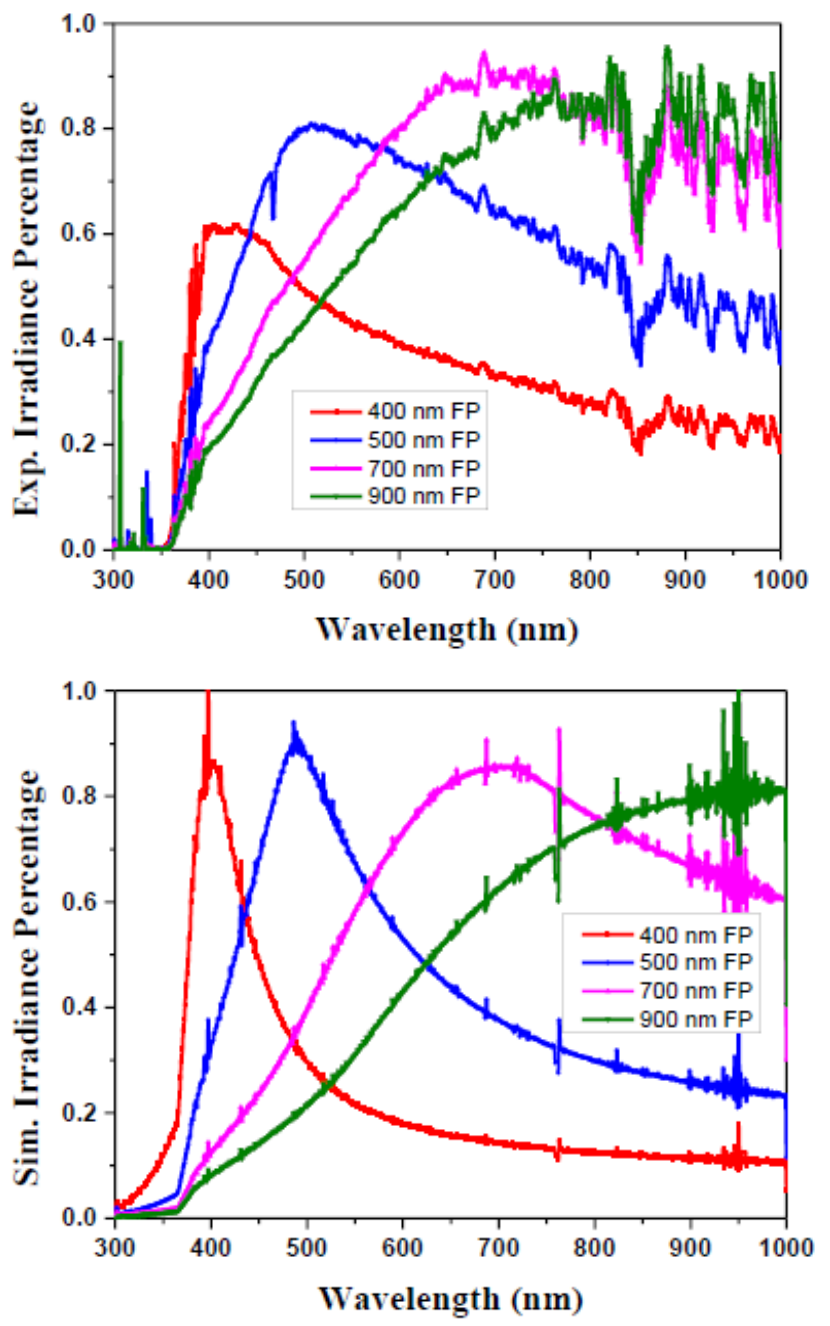

Figure 5: Aspheric lens characterization of (a) measured and ray-traced focal length versus wavelength, and (b) measured and (c) ray-traced spectra at sequential focal planes illustrating chromatic aberration. 


\section{REFERENCES}

[1] Wolf, M., "Limitations and possibilities for improvement of photovoltaic solar energy converters," Proc. IRE, 12461263 (1960).

[2] Blocker, W., "High-efficiency solar energy conversion through flux concentration and spectrum splitting," Proc. IEEE 66, 104-105 (1978).

[3] Polman, A., and Atwater, H. A., "Photonic design principles for ultrahigh-efficiency photovoltaics," Nat. Mat. 11, 174-177 (2012).

[4] Henry, C. H., "Limiting efficiencies of ideal single and multiple energy gap terrestrial solar cells," J. Appl. Phys. 51(8), 4494-4500 (1980).

[5] Torrey, E. R., Ruden, P. P., and Cohen, P. I., "Performance of a split-spectrum photovoltaic device operating under time-varying spectral conditions," J. Appl. Phys. 109, 074909 (2011).

[6] Barnett, A., et al., "Very high efficiency solar cell modules," Prog. Photovolt: Res. Appl. 17, 75-83 (2009).

[7] Russo, J. M., Zhang, D., Gordon, M., Vorndran, S. D., Wu, Y., and Kostuk, R. K., "Grating-over-lens concentrating photovoltaic spectrum splitting systems with volume holographic optical elements," Proc. SPIE 8821, 882106 (2013).

[8] Vlasov, A. S., et al., "Spectral-splitting concentrator photovoltaic modules based on $\mathrm{AlGaAs} / \mathrm{GaAs} / \mathrm{GaSb}$ and GaInP/InGaAs(P) solar cells," Technical Phys. 58(7), 1034-1038 (2013).

[9] Wang, P., Dominguez-Caballero, J. A., Friedman, D. J., and Menon, R., "A new class of multi-bandgap highefficiency photovoltaics enabled by broadband diffractive optics," Prog. Photovolt: Res. Appl., (2014). 